\title{
Endoscopic Decompression Can Be Effective for Diagnosing and Treating Tubercular Spondylodiskitis with Early Epidural Spinal Compression: A Retrospective Study of 18 Cases
}

\author{
Abhijit Pawar, Chirag Manwani, Raghavendra Thete, Mihir Bapat, Vishal Peshettiwar, Satishchandra Gore \\ Center for Bone and Joint, Kokilaben Dhirubhai Ambani Hospital and Medical Research Institute, Mumbai, India
}

\begin{abstract}
Study Design: Retrospective study.
Purpose: In this study, we describe an endoscopic method of effectively treating tubercular lumbar spondylodiskitis with early onset epidural spinal cord compression in the lumbar spine on magnetic resonance imaging (MRI).

Overview of Literature: Percutaneous aspiration and biopsy of spondylodiskitis under ultrasonography and computer tomography scan invariably provides an inadequate diagnosis.

Methods: From May 2015 to May 2017, 18 patients presented with intractable back pain and were diagnosed with tubercular spondylodiskitis on MRl; these patients were enrolled in this study. The goal was to confirm the pathogen on biopsy, drain the abscess, and perform debridement. Chemotherapy was started after histologic diagnosis, and data collected included blood cell counts, erythrocyte sedimentation rate, $\mathrm{C}$-reactive protein, and repeat MRI after 3 months.

Results: Mean duration of surgery was 52 minutes. Mean follow-up was 17 months. The average preoperative Visual Analog Scale score of 8 (range, 6-10) decreased to 3 (range, 1-8) postoperatively. Tubercular spondylodiskitis was observed in 14 cases; two cases were pyogenic, and the biopsy was inconclusive in two cases. After adequate chemotherapy, no recurrences were noted.

Conclusions: We hereby conclude that endoscopic biopsy and drainage can provide a better diagnosis and decrease pain in a predictable manner.
\end{abstract}

Keywords: Tubercular spondylodiskitis; Endoscopic debridement; Infective spondylodiskitis; Psoas abscess; Biopsy

\section{Introduction}

Spondylodiskitis is an infection involving the intervertebral discs, end plates, or vertebral body. Early diagnosis and prompt management is crucial for managing spinal infections [1-3]. Spinal tuberculosis (TB) is the most common extrapulmonary form of $\mathrm{TB}$, and it can cause severe pain, neuro deficits, and deformities [4]. Despite various treatment approaches, the management of spinal TB remains challenging. Tubercular spondylodiskitis is being diagnosed with increasing frequency due to greater availability of magnetic resonance imaging (MRI) [1]. Resistance to first-line antitubercular drugs has been observed in approximately $12 \%$ of cases [4]. Hence, biopsy

Received Sep 29, 2017; Revised Dec 5, 2017; Accepted Jan 9, 2018

Corresponding author: Abhijit Pawar

Center for Bone and Joint, Kokilaben Dhirubhai Ambani Hospital and Medical Research Institute, Andheri West, Mumbai 400053, India

Tel: +91-7387080391, Fax: +91-2230972030,E-mail: drabhijitpawar@gmail.com 
is crucial for confirming the diagnosis anddetermining patient sensitivity to antitubercular therapies. Percutaneous aspiration and biopsy under ultrasonography or computer tomography (CT) scan has a variable success rate of 36\%-91\% inpatients with infective spondylodiskitis [5-7]. Aspirates are often inadequate, and no organism is cultured. Percutaneous endoscopic discectomy was first used for the treatment of uncomplicated herniated discs in the early 1980s [8]. Minimally invasive management approaches are gradually gaining popularity due to their increased effectiveness and higher safety margins. Percutaneous endoscopic discectomy and debridement is a simple technique that is effective for debriding the disc space and features a diagnostic accuracy of $45 \%-87 \%$, according to the organism isolated [9-11]. With the rise in immunosuppressed patients due to human immunodeficiency virus or chronic diseases, the incidence of multidrug-resistant tubercular spondylodiskitis is increasing, and biopsies are required to diagnose and determine the sensitivity and resistance of the disease to various drugs [12]. We retrospectively evaluated 18 patients diagnosed with tubercular spondylodiskitis on MRI who were treated with endoscopic debridement and discectomy. This technique provides not only an adequate tissue sample but also significant symptom relief.

\section{Materials and Methods}

After approval of the Institutional Review Board of Kokilaben Dhirubhai Ambani Hospital, Mumbai (IRB approval no. KH0127), we enrolled 18 patients with intractable back pain, no neuro deficits, and early onset epidural spinal cord compression on lumbar spine MRI. Most of the patients complained of unremitting back pain, which disturbed their sleep and was not relieved by rest or analgesics. There were 12 females and six males with average age of 46 years, range 22-68 from May 2015 to May 2017. The inclusion criteria included a diagnosis of tubercular spondylodiskitis based on clinical examination and radiographic and MRI findings, and elevated erythrocyte sedimentation rate (ESR) and C-reactive protein (CRP). Patients with neuro deficits and spinal deformities were excluded from the study. Six patients had received antitubercular treatments (ATT) prior to the surgery and had experienced subsequent worsening of pain; consequently, ATT was stopped 1 week before endoscopy in these patients. The other 12 patients did not receive any antibiotics before surgery and until the biopsy results were available.

The surgical indication was intractable back pain and clinically worsening leg pain in patients who had not responded to chemotherapy and in whom MRI showed single-level spondylodiskitis with epidural spinal compression, a psoas abscess, or a paravertebral abscess. The goal of the surgery was to confirm the pathogen on biopsy and drain the abscess. Depending on each patient's symptoms and location of the abscess on MRI (right or left), the approach was decided.

Surgery was performed under local anesthesia and sedation, thereby making the procedure safe for high-risk patients. Under fluoroscopic guidance, the entry point was marked, and a long spinal needle was inserted in the affected disc space within the lumbar spine. The position of the guide wire was confirmed in both anterior-posterior and lateral views. The guide wire was passed, and the position confirmed. Stab incision was taken and, a dilator and canula were passed into the disc space (Fig. 1). A Karl Storz spinal endoscope was passed over the dilator into the appropriated disc space under $\mathrm{C}$-arm guidance, and a biopsy was obtained with punch forceps. Following this, the abscess was drained and wash given with normal saline.

Debridement was performed with discectomy forceps (Fig. 1). Decompression was performed until the dura and traversing nerve root were visualized. Because of the 12th rib, it is technically difficult to install a dilatation sleeve in the disc space at D12-L1; however, grasper forceps and an endoscopy hook can easily reach the disc space. The dilation sleeve and endoscopy sheath can be placed in the disc space between L2-S1. All specimens were sent for microscopy, genetic and histopathological diagnosis as well as culture. Normal saline was used for irrigation and debridement throughout the procedure. In each patient, more than enough tissue was obtained after biopsy without the fear of specimen dilution. Since the procedure was performed endoscopically without any bleeding, no drain was placed. No intraoperative or perioperative complications were observed in any of these cases. Postoperatively, the patients mobilized with either an LS belt or T-L Corset, depending on the location of the lesion. Postoperatively serial X-ray are taken for all the patients at 3-month intervals. In two patients, progression of the deformity was seen, and these two patients underwent subsequent surgery for stabilization of spine. Chemotherapy was started after diagnosis, and all the patients were monitored with blood cell counts, ESR, CRP, and repeat MRI after 3 months. 

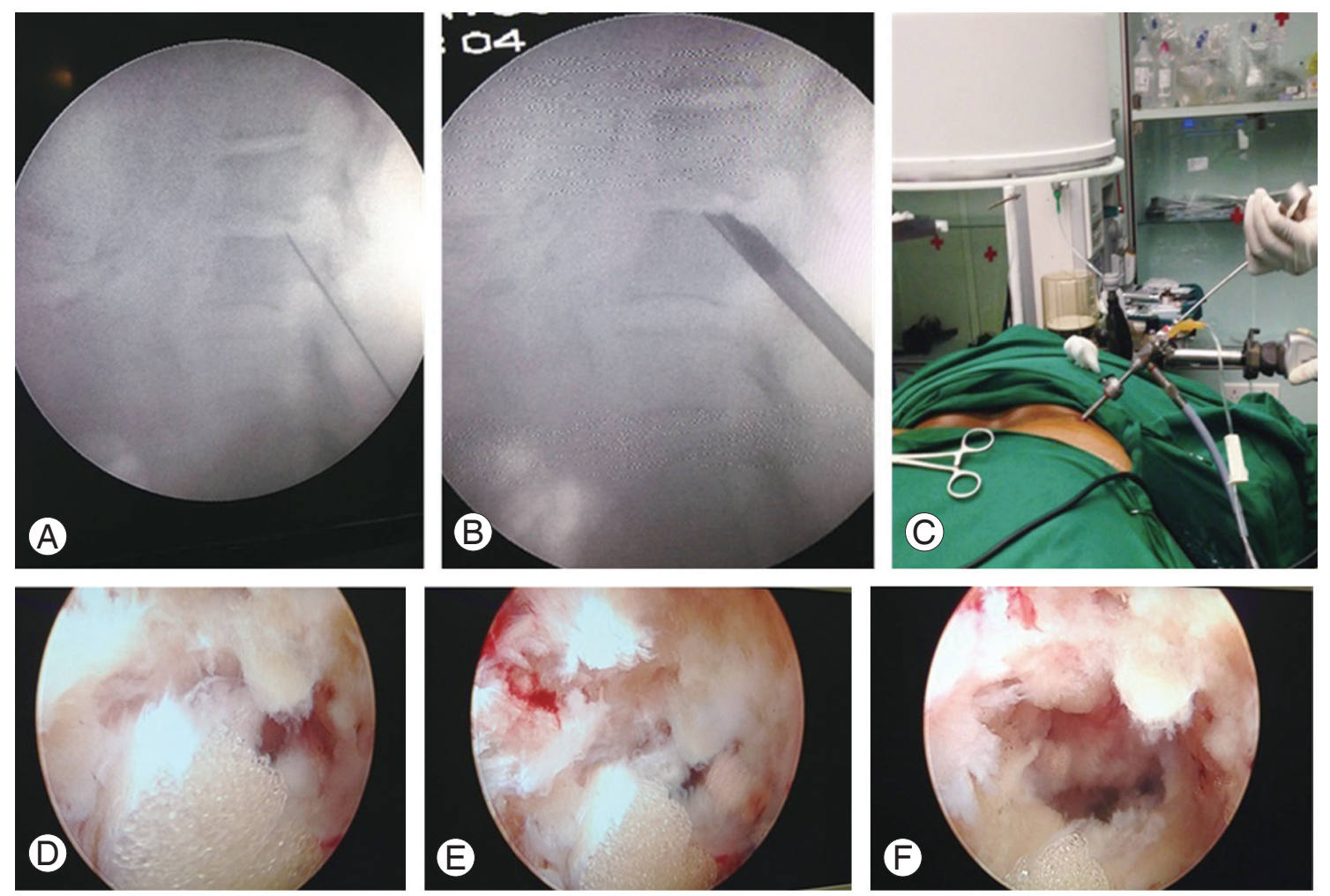

Fig. 1. (A-F) Technique of endoscopic decompression and debridement.

Table 1. Patients details and outcomes at 1 year

\begin{tabular}{|c|c|c|c|c|c|c|c|}
\hline No. & Age (yr) & Sex & $\begin{array}{c}\text { Site of } \\
\text { infection }\end{array}$ & Culture & $\begin{array}{l}\text { Preoperative } \\
\text { VAS score }\end{array}$ & $\begin{array}{l}\text { Postoperative VAS } \\
\text { score at } 3 \text { months }\end{array}$ & Outcome at 1 year \\
\hline 1 & 46 & $\mathrm{~F}$ & L1-L2 & M. tuberculosis & 8 & 3 & Healed with ATT \\
\hline 2 & 32 & $\mathrm{~F}$ & $\llcorner 4-5$ & S. aureus & 7 & 3 & Healed with antibiotics \\
\hline 3 & 22 & M & D12-L1 & M. tuberculosis & 9 & 4 & Healed with ATT \\
\hline 4 & 64 & M & $\mathrm{L} 1-\mathrm{L} 3$ & No growth & 6 & 2 & Healed on ATT \\
\hline 5 & 43 & $\mathrm{~F}$ & $\llcorner 4-5$ & M. tuberculosis & 7 & 2 & Healed on ATT \\
\hline 6 & 49 & M & D12-L1 & M. tuberculosis & 9 & 8 & Needed decompression and fusion \\
\hline 7 & 41 & $\mathrm{~F}$ & $\mathrm{~L} 1-\mathrm{L} 2$ & M. tuberculosis & 10 & 4 & Healed on ATT \\
\hline 8 & 61 & $\mathrm{~F}$ & L3-4 & M. tuberculosis & 7 & 2 & Healed on ATT \\
\hline 9 & 45 & M & L2-3 & M. tuberculosis & 7 & 0 & Healed on ATT \\
\hline 10 & 32 & M & L2-3 & M. tuberculosis & 8 & 1 & Healed on ATT \\
\hline 11 & 28 & $\mathrm{~F}$ & L2-3 & M. tuberculosis & 9 & 2 & Healed on ATT \\
\hline 12 & 65 & $\mathrm{~F}$ & L1-2 & M. tuberculosis & 10 & 3 & Healed on ATT \\
\hline 13 & 42 & $\mathrm{~F}$ & L1-2 & M. tuberculosis & 7 & 8 & Needed decompression and fusion \\
\hline 14 & 32 & $\mathrm{~F}$ & D12-L1 & M. tuberculosis & 8 & 1 & Healed on ATT \\
\hline 15 & 55 & M & $\llcorner 4-5$ & S. aureus & 9 & 2 & Healed with antibiotics \\
\hline 16 & 35 & $\mathrm{~F}$ & $\mathrm{~L} 2-3$ & M. tuberculosis & 7 & 1 & Healed on ATT \\
\hline 17 & 46 & M & D12-L1 & M. tuberculosis & 7 & 1 & Healed on ATT \\
\hline 18 & 54 & $\mathrm{~F}$ & L1-L2 & No growth & 6 & 1 & Healed on ATT \\
\hline
\end{tabular}

VAS, Visual Analog Scale; F, female; M, male; M. tuberculosis, Mycobacterium tuberculosis; S. aureus, Staphylococcus aureus. 


\section{Results}

Eighteen cases $(\mathrm{n}=18)$ with lumbar spondylodiskitis and abscesses on MRI were reviewed. There were 12 females and six males, with an average age of 46 years (range, 22-68 years), from May 2015 to May 2017. The mean duration of surgery was 52 minutes (range, 45-80 minutes). Mean follow-up duration was 17 months (range, 10-24 months). The average preoperative Visual Analog Scale (VAS) score was 8 (range, 6-10) but decreased to an average postoperative score of 3 (range, 1-8). Tubercular spondylodiskitis was observed in 14 cases; two cases were pyogenic, and the biopsy was inconclusive in two cases (Table 1).
Mycobacterium tuberculosis was isolated in 14 cases, Staphylococcus aureus in two, and no pathogen in two. We concluded that no organism was isolated in these two patients because they had already received ATT; hence, no organism could be isolated. Patients with tubercular spondylodiskitis were started on an antitubercular treatment for 1 year. The response to treatment was monitored with complete blood cell, ESR, and CRP, repeated every 3 months. MRIs were repeated at 3-4 months of follow-up visit and at 1 year after the completion of ATT. Two patients with who were reported at tubercular spondylodiskitis on MRI but microbiology and culture confirmed the presence of $S$. aureus. Both these patients responded to appropriate antibiotic therapies. Two patients complained
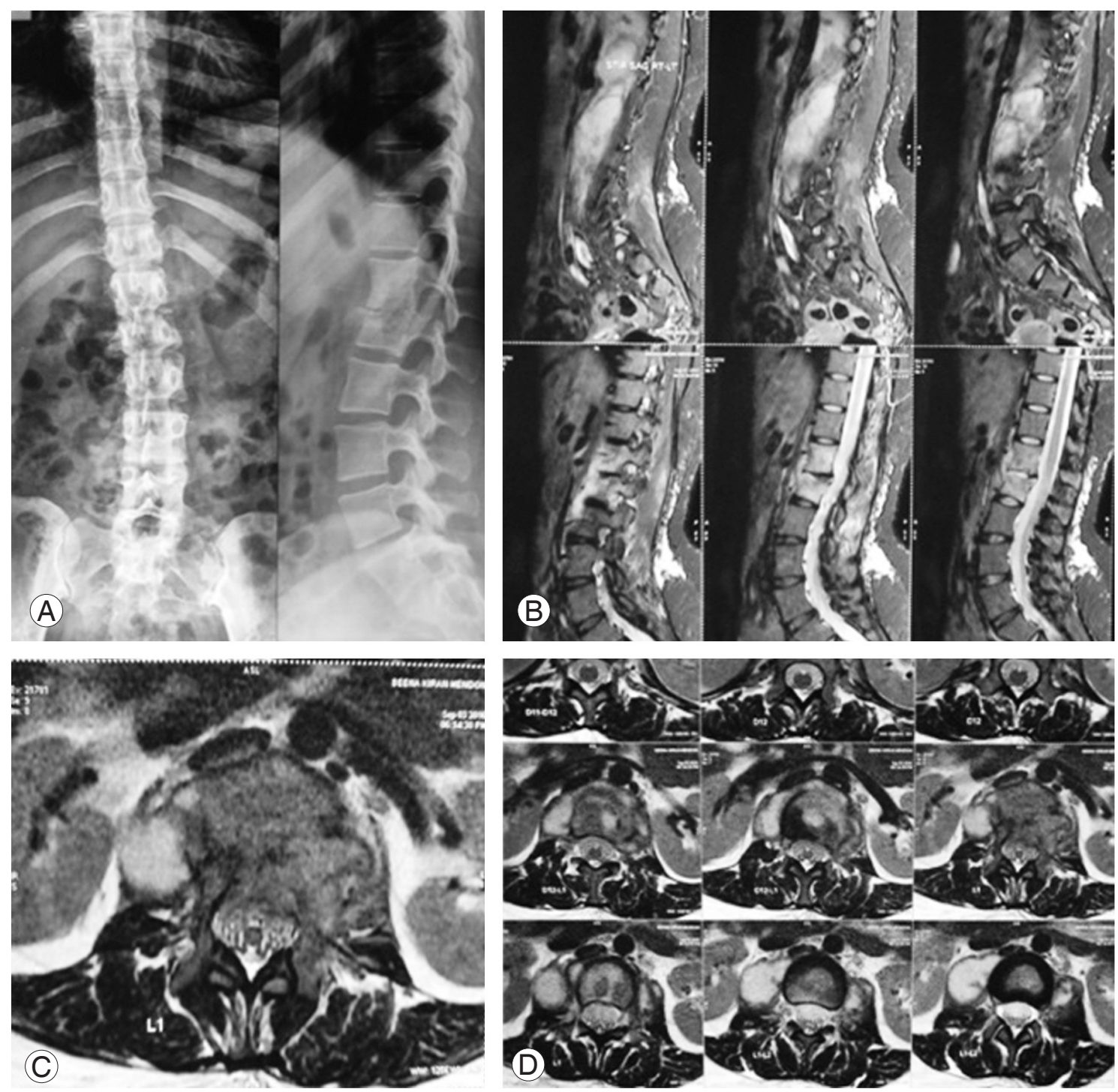

Fig. 2. (A-D) A 32-year-old female with D12-L1 spondylodiskitis with psoas abscess and disabling back pain. 

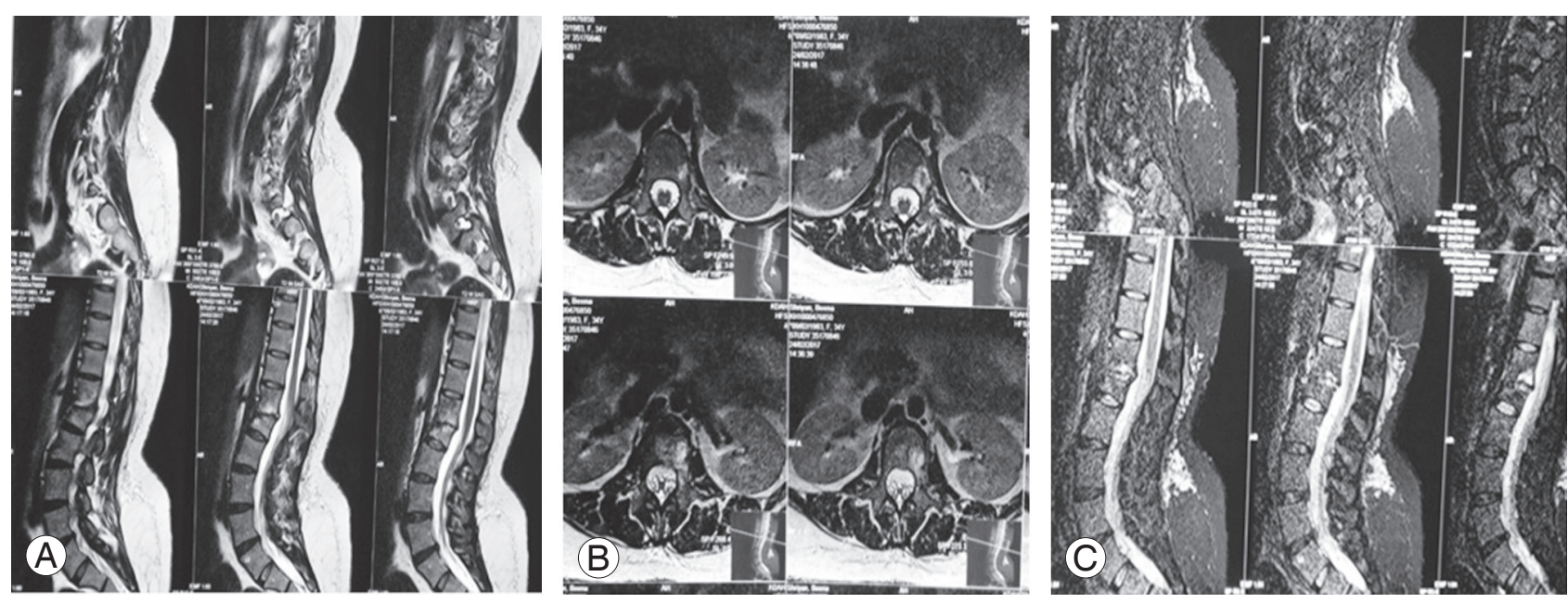

Fig. 3. (A-C) Postoperative magnetic resonance imaging at the 5-month follow-up with almost healed spondylodiskitis.

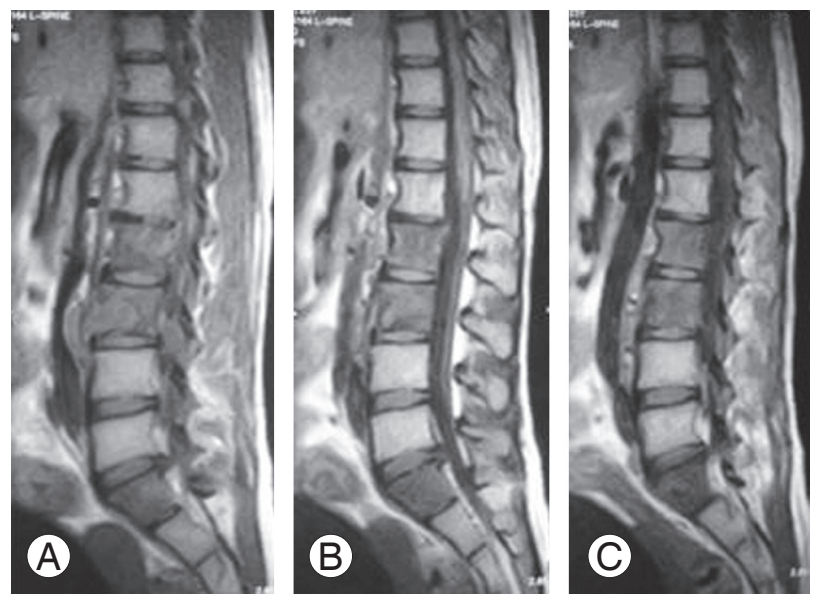

Fig. 4. (A-C) A 28-year-old female with intractable back and right leg pain with spondylodiskitis at $\mathrm{L} 2-\mathrm{L} 3$ and right psoas abscess.

of persistent back pain and leg pain after 1 year and underwent surgery for spinal stabilization.

\section{Discussion}

In this study, patients with thoracolumbar (TL) and lumbar tubercular spondylodiskitis, with epidural abscess and early spinal compression, were treated effectively with endoscopic decompression and debridement, followed by chemotherapy. Paravertebral and epidural abscesses were drained, focal tissues were debrided, and sclerotic bones were removed endoscopically. The procedures were performed under local anesthesia and sedation without any perioperative or postoperative complications. Sufficient material was obtained from the infected disc region, with a higher diagnostic accuracy (Figs. 2-5). Cultures were negative in two patients, despite having sufficient tissue for diagnosis. These two patients received ATT before endoscopy, and this could be the reason for the negative cultures. However, these patients later responded well to ATT because drug penetration is probably better after effective decompression. All the patients received ATT for 1 year post debridement. Two patients continued experiencing worsening back pain and leg pain at follow-up and underwent further decompression and instrumental fusion. MRIs of two patients detected paravertebral and epidural abscesses, and these patients were reported as having tubercular spondylodiskitis, but subsequent microbiology and cultures showed S. aureus. These patients responded to appropriate antibiotics for 6-8 weeks. Identifying the pathogen with biopsy is critical for the effective treatment of infectious spondylodiskitis $[5,13,14]$. The accuracy of CT-guided biopsy varies from 36\% to $91 \%$, according to various studies $[1,5,11,15]$. In a study conducted by Foreman et al. [15], CT-guided biopsy showed positive microbiology and histology results in only $28 \%$ of cases. Fouquet et al. [6] reported positive results in 36\% of cases (nine of 25 patients). Yang et al. [11] compared CTguided biopsy to endoscopic biopsy for infectious spondylodiskitis and experienced better results with endoscopy (90\% with endoscopy versus $47 \%$ with CT-guided procedures). The biopsy results were positive in 16 cases (89\%) in the present study. The average preoperative VAS score of 8 decreased to an average postoperative VAS score of 3. Endoscopic debridement, abscess drainage, inflammatory factor irrigation, and intradiscal pressure reduction 

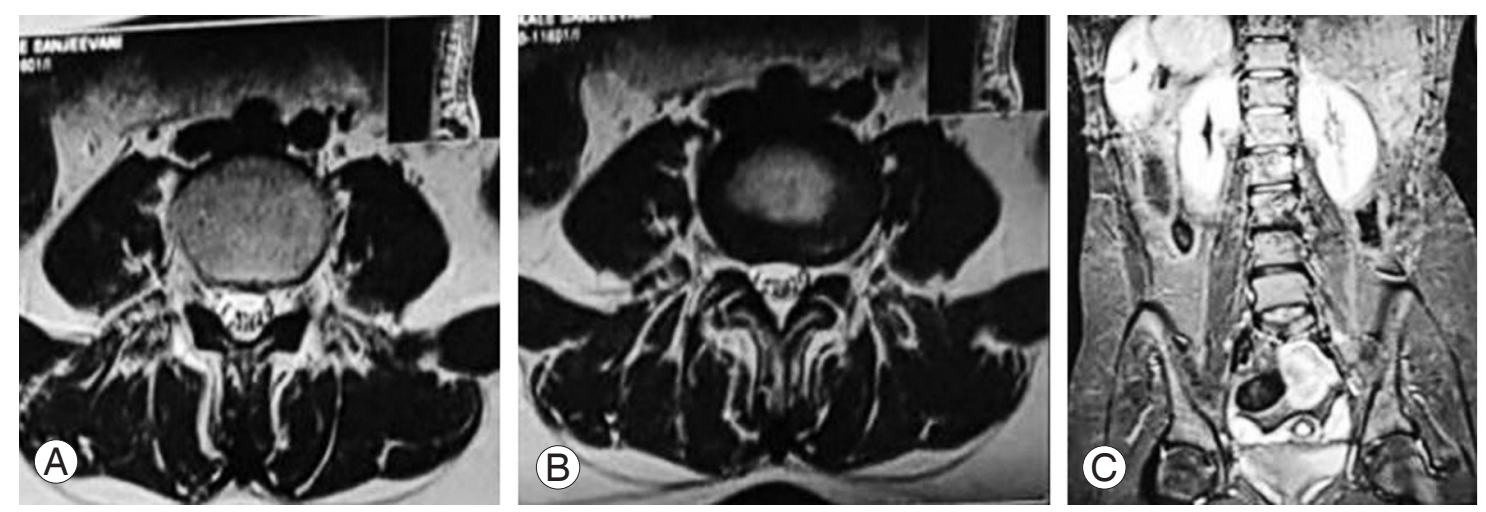

Fig. 5. (A-C) A 3-month postoperative magnetic resonance imaging after endoscopic debridement and discectomy.

contributed to the immediate and significant decrease in VAS scores. Fourteen patients diagnosed with M. tuberculosis on biopsy had normal MRIs and no back or leg pain at the 1-year follow-up. After the completion of ATT, two patients continued to experience worsening back and leg pain and were treated with posterior decompression and fusion.

With advancements in technology, CT-guided biopsy or ultrasound-guided biopsy is performed frequently in suspected cases $[2,11,16]$. Although the positive yield has increased, we still face issues of inconclusive results due to inadequate biopsy samples, inadequate symptom relief, and radiation exposure $[1,3,5,7]$. Although open biopsy has a higher rate of detection, it comes with its own set of problems, including the need for general anesthesia, which might be contraindicated inpatients with multiple comorbidities, increased risk of surgical site infection, and delayed recovery $[2,4,16]$. One of the biggest advantages of endoscopic biopsy and drainage is that along with obtaining adequate tissue sample, under direct visualization, abscess drainage facilitates symptom relief for patients $[9,17]$. Because the procedure is performed under local anesthesia and sedation, it can be performed even in high-risk patients. The rate of secondary surgical interventions can be reduced with adequate debridement and antitubercular therapies. Whether the duration of antibiotic therapy can be decreased using this technique still remains a question.

This study has several limitations. The retrospective nature of the study, small number of cases $(n=18)$, and short follow-up duration are among the most notable. A prospective study in a large population would validate the feasibility of this technique. Another limitation of this study is that this procedure was performed for spondylodiskitis of the lumbar spine and TL junction. It is techni- cally difficult to perform this technique at the craniovertebral junction and within the cervical spine. Although the TL junction and lumbar spine are the most commonly involved regions, with future advances, it might be possible to treat patients with cervical spine diskitis in a similar manner.

\section{Conclusions}

Endoscopic discectomy and debridement can be effective for the diagnosis of tubercular spondylodiskitis and can reduce pain in these patients. It can be an effective tool for treating elderly patients with multiple medical comorbidities as it can be performed under local anesthesia. The debridement of necrotic tissue is performed under direct visualization, and extensive anterior or posterior spinal decompression and instrumentation can be avoided. This technique can be an effective tool within the armamentarium of the spine surgeon for treating infective tubercular spondylodiskitis without deformities or neurological involvement.

\section{Conflict of Interest}

No potential conflict of interest relevant to this article was reported.

\section{References}

1. An HS, Seldomridge JA. Spinal infections: diagnostic tests and imaging studies. Clin Orthop Relat Res 2006;444:27-33.

2. Carragee EJ. Pyogenic vertebral osteomyelitis. J Bone Joint Surg Am 1997;79:874-80. 
3. Cottle L, Riordan T. Infectious spondylodiscitis. J Infect 2008;56:401-12.

4. Shetty AP, Viswanathan VK, Kanna RM, Shanmuganathan R. Tubercular spondylodiscitis in elderly is a more severe disease: a report of 66 consecutive patients. Eur Spine J 2017;26:3178-86.

5. Chew FS, Kline MJ. Diagnostic yield of CTguided percutaneous aspiration procedures in suspected spontaneous infectious diskitis. Radiology 2001;218:211-4.

6. Fouquet B, Goupille P, Jattiot F, et al. Discitis after lumbar disc surgery: features of "aseptic" and "septic" forms. Spine (Phila Pa 1976) 1992;17:356-8.

7. Rankine JJ, Barron DA, Robinson P, Millner PA, Dickson RA. Therapeutic impact of percutaneous spinal biopsy in spinal infection. Postgrad Med J 2004;80:607-9.

8. Kambin P, Schaffer JL. Percutaneous lumbar discectomy: review of 100 patients and current practice. Clin Orthop Relat Res 1989;(238):24-34.

9. Ito M, Abumi K, Kotani Y, Kadoya K, Minami A. Clinical outcome of posterolateral endoscopic surgery for pyogenic spondylodiscitis: results of $15 \mathrm{pa}$ tients with serious comorbid conditions. Spine (Phila Pa 1976) 2007;32:200-6.

10. Haaker RG, Senkal M, Kielich T, Kramer J. Percutaneous lumbar discectomy in the treatment of lumbar discitis. Eur Spine J 1997;6:98-101.
11. Yang SC, Fu TS, Chen LH, Chen WJ, Tu YK. Identifying pathogens of spondylodiscitis: percutaneous endoscopy or CT-guided biopsy. Clin Orthop Relat Res 2008;466:3086-92.

12. Kizilbash QF, Seaworth BJ. Multi-drug resistant tuberculous spondylitis: a review of the literature. Ann Thorac Med 2016;11:233-6.

13. Hadjipavlou AG, Katonis PK, Gaitanis IN, Muffoletto AJ, Tzermiadianos MN, Crow W. Percutaneous transpedicular discectomy and drainage in pyogenic spondylodiscitis. Eur Spine J 2004;13:707-13.

14. Urakov TM, Casabella AM, Levene HB. Percutaneous drainage of chronic destructive lumbar osteomyelitis abscess via the use of bilateral transpedicular trocar access. World Neurosurg 2016;92:583,e1-5.

15. Foreman SC, Schwaiger BJ, Gempt J, et al. MR and CT imaging to optimize CT-guided biopsies in suspected spondylodiscitis. World Neurosurg 2017;99:726-34.e7.

16. Grados F, Lescure FX, Senneville E, Flipo RM, Schmit JL, Fardellone P. Suggestions for managing pyogenic (non-tuberculous) discitis in adults. Joint Bone Spine 2007;74:133-9.

17. Cheng MT, Chang MC, Wang ST, Yu WK, Liu CL, Chen TH. Efficacy of dilute betadine solution irrigation in the prevention of postoperative infection of spinal surgery. Spine (Phila Pa 1976) 2005;30:168993. 